\title{
Differences in Evaluation of Hydroxychloroquine and Face Masks for SARS-CoV-2
}

\author{
Colin A. Ross* \\ Institute for Psychological Trauma, 1701 Gateway, Suite 349, Richardson, TX 75080, USA \\ ${ }^{\star}$ Corresponding author: Colin A. Ross, Institute for Psychological Trauma, 1701 Gateway, Suite 349, Richardson, TX 75080, USA
}

Received: December 15, 2020; Accepted: December 21, 2020; Published: December 31, 2020

\begin{abstract}
Current medical opinion, based on randomized controlled trials (RCTs), is that hydroxychloroquine is ineffective for treatment of SARS-CoV-2. Previous anecdotal and uncontrolled evidence that the drug might be helpful is now outweighed by RCTs. However, leading medical authorities and public health organizations such as the CDC, the Surgeon General and NIAID are strongly recommending wearing of face masks in public to reduce coronavirus transmission. Many governments and businesses are mandating face masks. These recommendations are based on weak, anecdotal, uncontrolled evidence and there are multiple meta-analyses of RCTs in the literature, not one of which found a single RCT in which face masks reduced viral transmission in public. The RCTs are ignored and not referenced on the CDC website. Organized medicine is taking the risk of serious blowback when and if the public learns that face masks are ineffective in viral pandemics. This blowback could undermine public confidence in vaccines and many other interventions and treatments for many different medical problems.
\end{abstract}

\section{Criteria Applied to Hydroxychloroquine for SARS- CoV-2}

In a recent opinion piece in $J A M A$, Saag [1] defined the criteria for evaluating scientific medical evidence, and specifically for evaluating potential interventions for treatment and prevention of coronavirus infections. His comments included the statement that: "First, a single report based on a small, nonrandomized study must be considered preliminary and hypothesis generating, not clinically actionable. Likewise, anecdotal case reports and case series that include several cases likewise must be considered anecdotal and preliminary." (p. 2162) These criteria are undisputed in medicine. They should be applied to all public health, pharmacological, vaccine and other preventive and treatment interventions for SARS-CoV-2. Saag applied these criteria in evaluating the effectiveness of hydroxychloroquine for the treatment of SARS-CoV-2 and concluded that: 1) based on the highest level of evidence, randomized controlled trials (RCTs), hydroxychloroquine is ineffective and should not be used, and 2) enthusiasm for hydroxychloroquine was not based on science or data, but instead was due to the politization of the pandemic: "However, the politicization of the treatment was a more important factor in promoting interest in use of this drug. On April 4, the US president, "speaking on gut instinct," promoted the drug as a potential treatment and authorized the US government to purchase and stockpile 29 million pills of hydroxychloroquine for use by patients with COVID-19. Of note, no health official in the US government endorsed use of hydroxychloroquine owing to the absence of robust data and concern about adverse effects." (p. 2162).

"The clear, unambiguous, and compelling lesson from the hydroxychloroquine story for the medical community and the public is that science and politics do not mix. Science, by definition, requires diligence and an honest assessment of findings; politics not so much. The number of articles in the peer-reviewed literature over the last several months that have consistently and convincingly demonstrated the lack of efficacy of a highly hyped "cure" for COVID-19 represent the consequence of the irresponsible infusion of politics into the world of scientific evidence and discourse. For other potential therapies or interventions for COVID-19 (or any other diseases), this should not happen again" (p. 2162). The present author is in agreement with these statements by Saag concerning hydroxychloroquine for treatment of SARS-CoV-2, and evaluation of any intervention for prevention or treatment of coronavirus infections. Presumably, the large majority of physicians are in agreement with Saag on these points. Initial hopefulness about hydroxychloroquine early in the pandemic was understandable, but it is now time to abandon that drug for that indication. Public health authorities such as the CDC, the NIAID and the U.S. Surgeon General are all in agreement on that point.

\section{Criteria Applied to Face Masks for SARS-CoV-2}

When we turn to the use of face masks for reducing coronavirus transmission in the community, a very different picture emerges. Now we see the CDC, NIAID and the Surgeon General strongly recommending the wearing of face masks in public, and we see governments and businesses mandating the wearing of face masks. This is said to be based on science and data. However, the evidence cited for the effectiveness of facemasks is anecdotal and uncontrolled. At the same time that face masks are being strongly recommended or mandated, five meta-analyses of RCTs for the use of face masks for reducing the transmission of viruses in public have not found a single RCT that showed any effect of face masks. This is why, in their 
December 1, 2020 Interim Guidance on mask use in the context of COVID-19, the World Health Organization [2] stated that: "At present there is only limited and inconsistent scientific evidence to support the effectiveness of masking of healthy people in the community to prevent infection with respiratory viruses, including SARS-CoV-2" (p. 8) In support of this conclusion, the World Health Organization referenced two recent papers published in the Annals of Internal Medicine, one of which was a randomized controlled trial of facemasks in Denmark with 4862 participants [3] that found no evidence of a protective effect of face masks. The second reference was to a review paper [4] of seven randomized controlled trials in the community and two in health care settings that found no protective effects of facemasks. Additional meta-analyses of RCTs for wearing of face masks in public include reviews of three RCTs [5], nine RCTs [6], four RCTs [7], ten RCTs [8] and most recently eleven RCTs [9]. The meta-analysis of eleven RCTs by Pezzolo et al. [9] involved a total of 7469 participants and found the relative risk for becoming corona virus-positive in people who wore face masks compared to people who did not to be 0.92 . They stated that this difference is not significant. Prior to April, 2020, the WHO, CDC, NIAID and Surgeon General were stating that there is no need to wear face masks in public to reduce transmission of any type of virus, and they had been saying so for years. Within a few months, in the United States but not in the WHO, a complete about face took place. This was justified as being based on newly emerging evidence, but in fact the new evidence was small, uncontrolled and anecdotal. As of December, 2020, the list of references on the CDC website used to justify public wearing of face masks for the COVID-19 pandemic is entirely anecdotal. Not one of the RCTs is referenced. Rather than the CDC basing its recommendation on RCTs, the RCTs are ignored.

An example of an anecdotal observational study referenced by the Director of the CDC [10], in a paper on which he is a coauthor, is a study of two coronavirus-positive salon workers who wore facemasks at work, as did 102 of their 104 exposed clients. None of the clients became ill, but none of them were tested for coronavirus so the number of asymptomatic carriers in the client group is unknown, therefore we can't reach any conclusion about the effectiveness of the face masks. In their paper, published in JAMA on July 14, 2020, the authors stated that, "At this critical juncture when COVID-19 is resurging, broad adoption of cloth face covering is a civic duty."

In the present climate, anyone questioning the effectiveness of face masks for preventing transmission of the coronavirus in public takes the risk of being attacked as a conspiracy theorist, a right-wing extremist, a racist, a white supremacist, a narcissist, or even as being brain damaged [11]. Writing in JAMA, Miller [11] offered possible explanations for science denial in the context of the SARS-CoV-2 pandemic, specifically denial that face masks are effective for reducing coronavirus transmission in public. He stated that, "The relationship between anti-science viewpoints and low science literacy underscores new findings regarding the brain mechanisms that form and maintain false beliefs." (p. 2255) Miller then went on to discuss how conspiracy theories that face masks do not work could be due to a variety of forms of neurological impairment including several different forms of dementia: "Conspiracy theories may bring security and calm, as with the patient with frontotemporal dementia who is content to believe they are rich." (p. 2256) Organized medicine has maintained a stance of being based on science and data, and it has stated that the wearing of face masks in public is proven by science, when in fact the opposite is true. There are more RCTs confirming that face masks do not work than there are RCTs confirming that hydroxychloroquine does not work.

\section{The Pore Size of Surgical Masks}

It is not physically possible for surgical masks to reduce transmission of the coronavirus by asymptomatic carriers. The size of the coronavirus is about 0.1 microns, and the size of respiratory aerosols is about 2-3 microns. The pore size of surgical masks is $50-100$ microns. Wearing a mask to prevent catching or transmitting the coronavirus is like putting a stake in the ground every 40 feet to prevent mice from coming onto your property [12-14]. Uninfected people and asymptomatic carriers are not coughing and sneezing in public, so they are not emitting any significant number of larger respiratory droplets. People who are symptomatic should stay at home. Isolation and quarantining should be the public health interventions for them. Face masks were never recommended for the flu because they don't work. Face masks for coronavirus are not based on science. They may be a symbol of solidarity, a social control mechanism, an anti-hysteria strategy, or a well-intentioned effort to help people feel safe. Whatever the motives of face mask advocates, face masks are not science or data-based and are not effective for reducing coronavirus transmission in public. The medical profession is taking the risk of future blowback and loss of confidence in all its public health recommendations, including vaccines, by insisting that doctor knows best concerning face masks.

\section{Conclusions}

Organized medicine and public health authorities have been stating for more than six months that face masks are effective for reducing coronavirus transmission in public. This is not scientifically true. If the criteria that are applied when evaluating hydroxychloroquine for COVID-19 were applied to face masks, the CDC, the Surgeon General and NIAID would be stating, as they did up till early 2020 , that there is no need to wear face masks in public.

\section{References}

1. Saag MS (2020) Misguided use of hydroxychloroquine for COVID-19: The Infusion of Politics Into Science. JAMA. [crossref]

2. World Health Organization (2020) Mask use in the context of COVID-19. Interim guidance, December 1, 2020

3. Bundgaard H, Bundgaard JS, Raaschou-Pedersen DET, Buchwald CV, Todsen T, et al. (2020) Effectiveness of adding a mask recommendation to other public health measures to prevent SARS-CoV-2 infection in Danish mask wearers: A randomized controlled trial. Annals of Internal Medicine. [crossref]

4. Chou R, Dana T, Jungbauer R, Weeks C, McDonagh MS (2020) Masks for prevention of respiratory virus infections, Including SARS-CoV-2, in health care and community settings: A living rapid review. Annals of Internal Medicine 173: 542-555. [crossref]

5. Brainard J, Jones N, Lake I, Hooper L, Hunter PR (2020) Face masks and similar barriers to prevent respiratory illness such as COVID-19: A rapid systematic review. Medrxiv.

6. Aggarwhal N, Dwarakananthan V, Gautham N, Ray A (2020) Facemasks for prevention of viral respiratory infections in community settings: A systematic review and meta-analysis. Indian Journal of Public Health 64: 192-200. [crossref]

7. Cowling BJ, Zhou Y, Ip DK, Leung GM, Aiello AE, et al. (2010) Face masks to prevent transmission of influenza virus: a systematic review. Epidemiology of Infection 138: 449-56. [crossref] 
Colin A. Ross (2020) Differences in Evaluation of Hydroxychloroquine and Face Masks for SARS-CoV-2

8. Xiao J, Shiv EYC, Gao H, Wong JY, Fong MW, et al. (2020) Nonpharmaceutical measures for pandemic influenza in nonhealthcare settings - personal protective and environmental measures. Emerging Infectious Diseases 26: 967-975.

9. Pezzolo E, Cazzaniga S, Gallus S, et al. (2020) Evidence from randomized controlled trials on the surgical masks' effect on the spread of respiratory infections in the community. Annals of Internal Medicine 26 November.

10. Brooks JT, Butler JC, Redfield RR (2020) Universal masking to prevent SAR-CoV-2 transmission - the time is now. JAMA. [crossref]
11. Miller BL. Science denial and COVID conspiracy theories (2020) Potential neurological mechanisms and possible responses. JAMA 324:2255-2256. [crossref]

12. Ross CA (2020) Thoughts on COVID-19. Journal of Neurology and Neurocritical Care 3: $1-3$.

13. Ross CA (2020) Facemasks are not effective for preventing transmission of the coronavirus. Journal of Neurology and Neurocritical Care 3: 1-2.

14. Ross CA (2020) How misinformation that facemasks are effective for reducing is transmitted. Journal of Neurology Neurocritical Care 3: 1-2. 